\title{
Mean-variance portfolio and contribution selection in stochastic pension funding
}

\author{
Ricardo Josa-Fombellida a, Juan Pablo Rincón-Zapatero ${ }^{\text {b,* }}$ \\ a Departamento de Estadística e Investigación Operativa, Universidad de Valladolid, Paseo Prado de la Magdalena, \\ s/n, 47005 Valladolid, Spain \\ b Departamento de Economía, Universidad Carlos III de Madrid, ClMadrid, 126-128, 28903 Getafe, Madrid, Spain
}

\begin{abstract}
In this paper we study the problem of simultaneous minimization of risks, and maximization of the terminal value of expected funds assets in a stochastic defined benefit aggregated pension plan. The risks considered are the solvency risk, measured as the variance of the terminal fund's level, and the contribution risk, in the form of a running cost associated to deviations from the evolution of the stochastic normal cost. The problem is formulated as a bi-objective stochastic problem of mean variance and it is solved with dynamic programming techniques. We find the efficient frontier and we show that the optimal portfolio depends linearly on the supplementary cost of the fund, plus an additional term due to the random evolution of benefits.
\end{abstract}

Keywords: Finance; Pension funding; Portfolio theory; Stochastic control; Mean-variance

\section{Introduction}

The optimal management of dynamic pension plans is an interesting problem due to the importance that pension funds have currently in financial markets, as well as their fundamental role in assuring the future wealth of participants in their retirement period.

Pension funds can be classified into the following two main categories: defined benefit (DB) pension plans and defined contribution (DC) pension plans. In a DB plan benefits are fixed in advance by the sponsor and contributions are designed to amortize the fund according to a previously chosen actuarial scheme. Future benefits due to participants are thus a liability for the sponsor, who bears the financial risk. Of course, this risk is increased with the formation of a risky portfolio that, however, offers higher expected returns, with the possibility then of reducing the amortization quote. It is the concern of the sponsor to drive the dynamic evolution of the fund having into account the trade off between risk and contribution. In a DC plan contri butions are fixed but benefits depend on the returns of the fund portfolio, so that the participants bear the risk.

\footnotetext{
* Corresponding author. Tel.: +34 91 6248666; fax: +34 916249329.

E-mail addresses: ricar@eio.uva.es (R. Josa-Fombellida), jrincon@eco.uc3m.es (J.P. Rincón-Zapatero).
} 
Beginning with the paper of Haberman and Sung (1994), DB plans have been usually modelled as linearquadratic optimal control problems. ${ }^{1}$ This is due to the fact that the dynamics of the fund is postulated linear, as in Merton's model, and that it is generally accepted that managers' objectives should be related with the minimization of solvency risk and contribution risk. These risk concepts are defined as quadratic deviations of fund wealth and amortization rates with respect to liabilities and normal cost, respectively. In an environment where liabilities are random, the risks so formulated do not correspond to the variance, which is by far the most common measure of risk. The aim of this paper is to study the optimal management of DB plans when the solvency risk is identified with the variance of the unfunded actuarial liability. To this end, the problem is settled in the familiar mean variance framework, translating the static model of Markowitz to the continuous-time setting of a DB plan that evolves with time.

Markowitz (1952) designed the mean variance model to compare securities and portfolios based in a tradeoff between their expected return and risk, measured as the variance of the return. From the point of view of optimization, the problem of portfolio selection is a multiobjective programming problem where it is desired to attain the highest possible expected return with the lowest possible variance. Since these objectives are in general mutually incompatible, the best can be done is to select portfolios where it is not possible to increase return without increasing risk, and reciprocally, where it is not possible to decrease risk without decreasing return. The set of pairs (return, variance) enjoying theses properties are called the Pareto frontier or efficient points set, and the associated portfolios are called efficient.

It has been several attempts in the literature to translate the mean variance methodology from the static case to the dynamic setting. The most successful and fundamental is of course the one initiated by Merton. It is worth noting, however, that Merton's model does not exactly fit the structure of the mean variance approach. It has been recently, in the papers by Zhou and $\mathrm{Li}$ (2000) and Li and $\mathrm{Ng}(2000)$ that the methodology has been more faithfully carried out to the dynamic setting, in continuous and in discrete time, respectively. In our paper we follow the formulation of Zhou and Li (2000) but with some modifications due to the specificities of a DB plan, as the inclusion of the supplementary cost as a control variable in addition to the quantities invested in the risky assets. This point is explained in Section 3 below. Moreover we use the Hamilton Jacobi Bellmam approach instead of the maximum principle.

Problems of mean variance type have been recently considered in pension plans from a static point of view in Colombo and Haberman (2005) and in Huang and Cairns (2005). A dynamic model for asset and liability management under the mean variance criteria has been studied in Chiu and Li (2006). The framework provided by these authors, although general, cannot be applied directly to a DB plan since several of the constitutive elements of the pension plan, as the amortization rate, normal cost, benefits and the technical actuarial rate, are not contemplated in the model. More fundamentally, a DB plan is identified by two different elements of control: investment decisions in the portfolio and amortization rate. The latter is absent in the framework provided by Chiu and $\mathrm{Li}$ (2006). The existence of an additional control variable requires a modification in the objective functional, introducing a running cost associated to the size of the amortization rate, more concretely, associated to quadratic deviations with respect to the stochastic normal cost. Thus our problem combines terminal payoffs due to the final levels of expected surplus/debt and of the variance of fund wealth (the stock variable) as well as an integral term or running cost that takes care of the contribution risk (the amortization rate is a flow variable).

Our paper follows Josa-Fombellida and Rincón-Zapatero (2004), where the benefits of the DB plan are stochastic, modelled by a geometric Brownian motion. Note that benefits is a non-tradable asset, hence the market is incomplete and, furthermore, we also consider the existence of correlation between the sources of uncertainty in the benefits and in the asset returns.

The paper is organized as follows. Section 2 defines the elements of the pension scheme and describes the financial market where the fund operates. Section 3 is devoted to formulate the management of the DB plan in a mean variance framework, with the simultaneous objectives of minimizing the expected unfunded actuarial liability, as well as its variance at the final time, and to minimize the contribution rate risk over the planning

\footnotetext{
${ }^{1}$ There is a growing amount of papers devoted to the optimal management of DB pension plans (see e.g. Haberman and Sung, 1994; Chang, 1999; Cairns, 2000; Haberman et al., 2000; Taylor, 2002; Chang et al., 2002; Josa-Fombellida and Rincón-Zapatero, 2001, 2004, 2006a,b).
} 
interval. The problem is solved in Section 4 using first the well known scalarization method and then the device provided in Zhou and $\mathrm{Li}$ (2000). Once the Pareto frontier is obtained, we compute the total expected supplementary cost and the total expected contribution rate. Section 5 serves as a numerical illustration of previous results. Finally, Section 6 is dedicated to establishing some conclusions. All proofs are developed in Appendix A.

\section{The pension model}

Consider a DB pension plan of aggregated type where at every instant of time active participants as well as retired participants coexist. Benefits payed to the participants at the age of retirement are fixed in advance by the sponsor and are governed by an exogenous process which source of randomness is correlated with the financial market. To cover the liabilities in an efficient way, the manager creates a portfolio and design an amortization scheme varying with time. The main elements intervening in a DB plan are the following.

$T$ planning horizon or date of the end of the pension plan, with $0<T<\infty$,

$F(t) \quad$ value of fund assets at time $t$,

$P(t) \quad$ benefits promised to the participants at time $t$. They are related with the salary at the moment of retirement,

$C(t) \quad$ contribution rate made by the sponsor at time $t$ to the funding process,

$A L(t)$ actuarial liability at time $t$, that is, total liabilities of the sponsor,

$N C(t)$ normal cost at time $t$; if the fund assets match the actuarial liability, and if there are no uncertain elements in the plan, the normal cost is the value of the contributions allowing equality between asset funds and liabilities,

$U A L(t)$ unfunded actuarial liability at time $t$, equal to $A L(t)-F(t)$,

$S C(t) \quad$ supplementary cost at time $t$, equal to $C(t)-N C(t)$,

$\delta \quad$ constant rate of valuation of the liabilities, which can be specified by the regulatory authorities.

\subsection{The actuarial functions}

Following Josa-Fombellida and Rincón-Zapatero (2004) we suppose that disturbances there exist affecting the evolution of benefits and hence the evolution of the normal cost and the actuarial liability. To model this randomness, we consider a probability space $(\Omega, \mathscr{F}, \mathbb{P})$, where $\mathscr{F}=\left\{\mathscr{F}_{t}\right\}_{t \geqslant 0}$ is a complete and right continuous filtration generated by the one-dimensional Brownian motion $\{B(t)\}_{t \geqslant 0}$ and $\mathbb{P}$ is a probability measure on $\Omega$. The more general case is to suppose benefits $P$ is a diffusion process built from $B$, that is, $P$ satisfies the stochastic differential equation (SDE)

$$
\mathrm{d} P(t)=\kappa(t, P(t)) \mathrm{d} t+\eta(t, P(t)) \mathrm{d} B(t), \quad 0 \leqslant t \leqslant T, P(0)=P_{0},
$$

where $P_{0}$ is the value of the initial liabilities, and where $\kappa$ and $\eta$ are functions such that the SDE has a unique solution. For analytical tractability, we will need a more concrete specification for benefits, $P$. We will suppose that benefits follows a geometric Brownian motion. It is the natural extension of the deterministic case where $P$ is an exponential function (see Bowers et al., 1986). This assumption is natural since in general benefits depends on salary and population plan, which show in the average exponential growth subject to random disturbances that may supposed to be proportional to the variables' size.

Assumption 1. The benefits $P$ satisfies

$$
\mathrm{d} P(t)=\kappa P(t) \mathrm{d} t+\eta P(t) \mathrm{d} B(t), \quad t \geqslant 0,
$$

where $\kappa \in \mathbb{R}$ and $\eta \in \mathbb{R}_{+}$. The initial condition $P(0)=P_{0}$ is a random variable that represents the initial liabilities.

To compute the actuarial functions $A L$ and $N C$, we suppose that all information accumulated up to time $t$ is used, under the real probability measure $\mathbb{P}$. The definitions of actuarial liability and normal cost, given in Josa-Fombellida and Rincón-Zapatero (2004), extend to the stochastic case these concepts from Bowers et al. (1986) as follows: 


$$
\begin{aligned}
& A L(t)=\int_{a}^{d} \mathrm{e}^{-\delta(d-x)} M(x) \mathbb{E}\left(P(t+d-x) \mid \mathscr{F}_{t}\right) \mathrm{d} x, \\
& N C(t)=\int_{a}^{d} \mathrm{e}^{-\delta(d-x)} m(x) \mathbb{E}\left(P(t+d-x) \mid \mathscr{F}_{t}\right) \mathrm{d} x,
\end{aligned}
$$

for every $t \geqslant 0$, where $\mathbb{E}\left(\cdot \mid \mathscr{F}_{t}\right)$ denotes conditional expectation with respect to $\mathscr{F}_{t}$, and where $M(x)$ is a distribution function representing the percentage of actuarial value of future benefits accumulated until age $x$, and where $m(x)$ is its associated density function. Without lost of generality we are supposing that all members enter into plan at age $a$ and retire at the common age $d$. Thus, to compute the actuarial functions at time $t$, the manager makes use of the information available up to that time, in terms of the conditional expectation. This procedure, instead of looking for a risk-neutral probability measure and then to compute the conditional expectation under this measure, is justified since the liabilities are non-tradeable in the financial market, thus the inherent risk cannot be hedged. The behavior of the actuarial functions $A L$ and $N C$ are then given in the following result, see Proposition 2.1 in Josa-Fombellida and Rincón-Zapatero (2004).

Proposition 2.1. Under Assumption 1 there are constants $\psi_{A L}$ and $\psi_{N C}$ such that $A L=\psi_{A L} P$ and $N_{C}=\psi_{N C} P$. Furthermore, $\psi_{N C}=1+(\kappa-\delta) \psi_{A L}$ and the identity $N C(t)-P(t)=(\kappa-\delta) A L(t)$ holds for every $t \geqslant 0$.

From this proposition we deduce:

$$
\mathrm{d} A L(t)=\kappa A L(t) \mathrm{d} t+\eta A L(t) \mathrm{d} B(t), \quad A L(0)=\psi_{A L} P_{0}
$$

and also

$$
\mathrm{d} N C(t)=\kappa N C(t) \mathrm{d} t+\eta N C(t) \mathrm{d} B(t), \quad N C(0)=\psi_{N C} P_{0} .
$$

We will denote by $A L_{0}$ and $N C_{0}$ the initial values of the actuarial liability and the normal cost, respectively, that is $A L_{0}=\psi_{A L} P_{0}$ and $N C_{0}=\psi_{N C} P_{0}$.

\subsection{The financial market}

In the rest of this section we describe the financial market where the fund operates. Given an $(n+1)$-dimensional standard Brownian motion $\left(w_{0}, w_{1}, \ldots, w_{n}\right)^{\top}$, we consider the complete probability space $(\Omega, \mathscr{G}, \mathbb{P})$ generated by it, that is to say, $\mathscr{G}$ is the filtration $\left\{\mathscr{G}_{t}\right\}_{t \geqslant 0}$, with $\mathscr{G}_{t}=\sigma\left\{w_{0}(s), w_{1}(s), \ldots, w_{n}(s) ; 0 \leqslant s \leqslant t\right\}$.

The plan sponsor manages the fund in the planning interval $[0, T]$ by means of a portfolio formed by $n$ risky assets $\left\{S^{i}\right\}_{i 1}^{n}$, which are correlated geometric Brownian motions, generated by $w=\left(w_{1}, \ldots, w_{n}\right)^{\top}$, and a riskless asset $S^{0}$, as proposed in Merton (1971), that is, whose evolutions are given by the equations:

$$
\begin{aligned}
& \mathrm{d} S^{0}(t)=r S^{0}(t) \mathrm{d} t, \quad S^{0}(0)=1, \\
& \mathrm{~d} S^{i}(t)=S^{i}(t)\left(b_{i} \mathrm{~d} t+\sum_{j}^{n} \sigma_{i j} \mathrm{~d} w_{j}(t)\right), \quad S^{i}(0)=s_{i}>0, i=1,2, \ldots, n .
\end{aligned}
$$

Here $r>0$ denote the short risk-free rate of interest, $b_{i}>0$ the mean rate of return of the $i$ th risky asset and $\sigma_{i j} \geqslant 0$ the covariance between asset $i$ and $j$, for all $i, j=1, \ldots, n$. It is assumed $b_{i}>r$ for all $i$, so the sponsor has incentives to invest with risk. We suppose that there exists correlation $q_{i} \in[-1,1]$ between $B$ and $w_{i}$, for $i=1, \ldots, n$. As a consequence, $B$ is expressed in terms of $\left\{w_{i}\right\}_{i 0}^{n}$ as $B(t)=\sqrt{ } 1-q^{\top} q w_{0}(t)+q^{\top} w(t)$, where $q^{\top} q \leqslant 1$ for $q=\left(q_{1}, q_{2}, \ldots, q_{n}\right)^{\top}$. In this way the influence of salary and inflation in the evolution of liabilities $P$ is taken into account, as well as the effect of inflation on the prices of the assets.

The amount of fund invested in time $t$ in the risky asset $S^{i}$ is denoted by $\lambda_{i}(t), i=1,2, \ldots, n$. The remainder, $F(t)-\sum_{i}^{n} \lambda_{i}(t)$, is invested in the bond. Borrowing and shortselling is allowed. A negative value of $\lambda_{i}$ means that the sponsor sells a part of his risky asset $S^{i}$ short while, if $\sum_{i{ }_{1}}^{n} \lambda_{i}$ is larger than $F$, then he or she gets into debt to purchase the stocks, borrowing at the riskless interest rate $r$. We suppose the investment strategy $\{\boldsymbol{\Lambda}(t): t \geqslant 0\}$, with $\boldsymbol{\Lambda}(t)=\left(\lambda_{1}(t), \lambda_{2}(t), \ldots, \lambda_{n}(t)\right)^{\top}$, is a control process adapted to filtration $\left\{\mathscr{G}_{t}\right\}_{t \geqslant 0}, \mathscr{G}_{t}$-measurable, Markovian and stationary, satisfying 


$$
\mathbb{E} \int_{0}^{T} \boldsymbol{\Lambda}(t)^{\top} \boldsymbol{\Lambda}(t) \mathrm{d} t<\infty
$$

where $\mathbb{E}$ is the expectation operator. The contribution rate process $C(t)$ is also an adapted process with respect to $\left\{\mathscr{G}_{t}\right\}_{t \geqslant 0}$ verifying

$$
\mathbb{E} \int_{0}^{T} S C^{2}(t) \mathrm{d} t<\infty
$$

Therefore, the fund dynamic evolution under the investment policy $\boldsymbol{\Lambda}$ is $^{2}$

$$
\mathrm{d} F(t)=\sum_{i=1}^{n} \lambda_{i}(t) \frac{\mathrm{d} S^{i}(t)}{S^{i}(t)}+\left(F(t)-\sum_{i=1}^{n} \lambda_{i}(t)\right) \frac{\mathrm{d} S^{0}(t)}{S^{0}(t)}+(C(t)-P(t)) \mathrm{d} t .
$$

By substituting (2) and (3) in (6), we obtain:

$$
\mathrm{d} F(t)=\left(r F(t)+\sum_{i}^{n} \lambda_{i}(t)\left(b_{i}-r\right)+C(t)-P(t)\right) \mathrm{d} t+\sum_{i}^{n} \sum_{j}^{n} \lambda_{i}(t) \sigma_{i j} \mathrm{~d} w_{j}(t),
$$

with initial condition $F(0)=F_{0}>0$.

Next we will assume the matrix notation: $\sigma=\left(\sigma_{i j}\right), \mathbf{b}=\left(b_{1}, b_{2}, \ldots, b_{n}\right)^{\top}, \mathbf{1}=(1,1, \ldots, 1)^{\top}$ and $\Sigma=\sigma \sigma^{\top}$. We take as given the existence of $\Sigma^{-1}$, that is to say, $\sigma^{-1}$. Finally the vector of standardized risk premia or Sharpe ratio of the portfolio is denoted by $\theta=\sigma^{-1}(\mathbf{b}-r \mathbf{1})$. So, we can write (7) as

$$
\mathrm{d} F(t)=\left(r F(t)+\boldsymbol{\Lambda}^{\top}(t)(\mathbf{b}-r \mathbf{1})+C(t)-P(t)\right) \mathrm{d} t+\boldsymbol{\Lambda}^{\top}(t) \sigma \mathrm{d} w(t),
$$

that, with the initial condition $F(0)=F_{0}$, determines the fund evolution.

We assume throughout the paper, as in Josa-Fombellida and Rincón-Zapatero (2004), that the technical interest rate coincides with the rate of return of the bond plus an additional term related with the market risk of the liabilities. In fact, this definition of $\delta$ adjusts the risk of the discounted future value of the liabilities, as if the preferences of the sponsor were risk-neutral. We are using here the equilibrium approach of Constantinides (1978), as it is detailed in Appendix A. On the other hand, this value of $\delta$ allows us to obtain the optimal contribution and portfolio in explicit form.

Assumption 2. The technical rate of actualization is $\delta=r+\eta q^{\top} \theta$.

Notice that if either benefits are deterministic or there is no correlation between benefits and the financial market, then $\delta$ is the risk-free rate of interest. With positive (resp. negative) correlation, the valuation of liabilities is $r$ plus a positive (resp. negative) term, weighted by the product of the instantaneous variance of $P$ and the Sharpe ratio of the assets. This is the right way to price liabilities, since with positive (resp. negative) correlation it is expected that liabilities and assets move in the same (resp. opposite) direction.

Assumption 2 allows us to write $\operatorname{SDE}(8)$ in terms of $X=-U A L=F-A L$ and of $S C=C-N C$ as

$$
\begin{aligned}
d X(t)= & \left(r X(t)+\boldsymbol{\Lambda}^{\top}(t)(\mathbf{b}-r \mathbf{1})+S C(t)-\eta q^{\top} \theta A L(t)\right) \mathrm{d} t-\eta A L(t) \sqrt{ } 1-q^{\top} q \mathrm{~d} w_{0}(t) \\
& +\left(\boldsymbol{\Lambda}^{\top}(t) \sigma-\eta A L(t) q^{\top}\right) \mathrm{d} w(t),
\end{aligned}
$$

with the initial condition $X(0)=X_{0}=F_{0}-A L_{0}$. See Appendix A.

To fix the nomenclature, we will suppose along the paper that the fund is underfunded at time $0, X_{0}<0$, so that $X$ has the meaning of debt. The same interpretation of the results are valid when the fund is overfunded, but then $X$ is surplus.

\section{The problem formulation}

The objective of the manager is double. On the one hand, it is to minimize the expected unfunded actuarial liability $\mathbb{E} U A L(T)=-\mathbb{E} X(T)=-(\mathbb{E} F(T)-\mathbb{E} A L(T))$, or equivalently to maximize the expected value of fund's

\footnotetext{
${ }^{2}$ This is the familiar equation obtained and justified in e.g. Merton (1990, p. 124). The only difference is that consumption is replaced here by $P \quad C$.
} 
assets. Note that as we are supposing $X<0$, most often we refer to $X$ as debt. On the other hand, the aim is to minimize the variance of the terminal debt, $\operatorname{Var} X(T)$, and the contribution risk $S C^{2}$ on the interval $[0, T]$. This bi-objective problem reflects the concern of the promoter of increase fund assets to pay due benefits, but at the same time not subject the pension fund to large variations to provide stability to the plan. Minimization of the contribution risk (related with the security of plan) has been considered in other works as Haberman and Sung (1994), Haberman et al. (2000) and Josa-Fombellida and Rincón-Zapatero (2001, 2004).

Thus we are considering a multiobjective optimization problem with two criteria ${ }^{3}$

$$
\min _{(S C, \boldsymbol{\Lambda}) \in \mathscr{A}_{X_{0}, A L_{0}}}\left(J_{1}(S C, \boldsymbol{\Lambda}), J_{2}(S C, \boldsymbol{\Lambda})\right) \doteq \min _{(S C, \boldsymbol{\Lambda}) \in \mathscr{A}_{X_{0}, A L_{0}}}\left(-\mathbb{E} X(T), \mathbb{E} \int_{0}^{T} S C^{2}(t) \mathrm{d} t+\mathbb{V} \operatorname{ar} X(T)\right),
$$

subject to (9), (1). Here $\mathscr{A}_{X_{0}, A L_{0}}$ is the set of measurable processes $(S C, \boldsymbol{\Lambda})$, where $S C$ satisfies (5), $\boldsymbol{\Lambda}$ satisfies (4) and such that (1) and (9) admit a unique solution $\mathscr{G}_{t}$-measurable adapted to the filter $\left\{\mathscr{G}_{t}\right\}_{t \geqslant 0}$.

Note that problem (1), (9), (10) is a mean variance problem similar to the one studied in Zhou and $\mathrm{Li}$ (2000), but with the additional control variable $S C$ in the state Eq. (9), and an additional running cost in (10).

An admissible control process $\left(S C^{*}, \boldsymbol{\Lambda}^{*}\right)$ is Pareto efficient (or simply efficient) if there exists no admissible $(S C, \boldsymbol{\Lambda})$ such that

$$
J_{1}(S C, \boldsymbol{\Lambda}) \leqslant J_{1}\left(S C^{*}, \boldsymbol{\Lambda}^{*}\right), \quad J_{2}(S C, \boldsymbol{\Lambda}) \leqslant J_{2}\left(S C^{*}, \boldsymbol{\Lambda}^{*}\right),
$$

with at least one of the inequalities being strict. The pairs $\left(J_{1}\left(S C^{*}, \boldsymbol{\Lambda}^{*}\right), J_{2}\left(S C^{*}, \boldsymbol{\Lambda}^{*}\right)\right) \in \mathbb{R}^{2}$ form the Pareto frontier. We will call to $S C^{*}$ an efficient supplementary cost, $C^{*}=S C^{*}+N C$ an efficient contribution rate and $\boldsymbol{\Lambda}^{*}$ an efficient portfolio. Throughout the text the term optimal must be understood in the sense of efficiency. Actually, we are not interested in the representation and properties of the Pareto frontier, but in the pairs $(-\mathbb{E} X(T), \operatorname{Var} X(T))$ for optimal $X(T)$, that we call the mean variance efficient frontier.

According to Da Cunha and Polak (1967) when the objective functionals defining the multiobjective program are convex, the Pareto optimal points can be found solving a scalar optimal control problem where the dynamics remain the same and where the objective functional is a convex combination of the original cost functionals. In our case Eqs. (1), (9) are linear, so both $J_{1}$ and $J_{2}$ are obviously convex. Therefore, the original problem (1), (9), (10) is equivalent to the scalar problem

$$
\min _{(S C, \boldsymbol{\Lambda}) \in \mathscr{A}_{X_{0}, A L_{0}}} J_{1}(S C, \boldsymbol{\Lambda})+\mu J_{2}(S C, \boldsymbol{\Lambda})=\min _{(S C, \boldsymbol{\Lambda}) \in \mathscr{A} X_{0}, A L_{0}}-\mathbb{E} X(T)+\mu\left(\mathbb{E} \int_{0}^{T} S C^{2}(t) \mathrm{d} t+\mathbb{V} \operatorname{ar} X(T)\right),
$$

subject to (1), (9), with $\mu>0$ a weight parameter. As $\mu$ varies in the interval $(0, \infty)$, the solutions of (11) describe the Pareto frontier. Notice that $\mu$ serves the manager to transfer linearly units of risk to units of expected return, and reciprocally. The size of $\mu$ indicates which one of the objectives is of more concern for the manager, to reduce risk or to reduce debt.

Problem (1), (9), (11) is not a standard stochastic optimal problem due to the term $(\mathbb{E} X(T))^{2}$ in the variance, and the dynamic programming approach cannot be applied here. Following Zhou and $\mathrm{Li}(2000)$ or $\mathrm{Li}$ and $\mathrm{Ng}$ (2000) we propose an auxiliary problem that turns out to be a stochastic problem of linear-quadratic type:

$$
\min _{(S C, \boldsymbol{\Lambda}) \in \mathscr{A}_{X_{0}, A L_{0}}} J(S C, \boldsymbol{\Lambda}) \doteq \min _{(S C, \mathbf{\Lambda}) \in \mathscr{A}_{X_{0}, A L_{0}}} \mathbb{E} \int_{0}^{T} S C^{2}(t) \mathrm{d} t+\mathbb{E}\left(X^{2}(T)-2 \gamma X(T)\right),
$$

subject to (1), (9), where $\gamma \in \mathbb{R}$.

The relationship between problems (1), (9), (11) and (1), (9), (12) is shown in the following result.

Proposition 3.1. For any $\mu>0$, if $\left(S C^{*}, \Lambda^{*}\right)$ is an optimal control of (1), (9), (11) with associated optimal debt $X^{*}$, then it is an optimal control of (1), (9), (12) for $\gamma=(2 \mu)^{1}+\mathbb{E} X^{*}(T)$.

The main consequence of Proposition 3.1 is that any optimal solution of problem (1), (9), (11) can be found solving problem (1), (9), (12). This will be done in the following section.

\footnotetext{
${ }^{3}$ The complete notation for the objective functionals would be $J_{1}((t, x, y) ;(S C, \boldsymbol{\Lambda}))=\mathbb{E}_{t x y} X(T)=\mathbb{E}(X(T) \mid X(t)=x, A L(t)=y)$ and $J_{2}((t, x, y) ;(S C, \boldsymbol{\Lambda}))=\mathbb{E}_{t x y} \int_{t}^{T} S C^{2}(s) \mathrm{d} s+\mathbb{V a r}_{t x y} X(T)$.
} 


\section{Optimal contributions, optimal portfolio and the efficient frontier}

In this section we find the efficient frontier for the original problem (1), (9), (10). Previously we solve the problem (1), (9), (12), depending on the parameter $\gamma$.

Theorem 4.1. The optimal rate of supplementary cost and the optimal investment in the risky assets are given by

$$
\begin{aligned}
& S C^{*}(t, X, A L)=f(t)\left(\gamma \mathrm{e}^{-r(T-t)}-X\right), \\
& \boldsymbol{\Lambda}^{*}(t, X, A L)=\Sigma^{-1}(\mathbf{b}-r \mathbf{1})\left(\gamma \mathrm{e}^{-r(T-t)}-X\right)+\eta \sigma^{-\top} q A L,
\end{aligned}
$$

where

$$
f(t)=\frac{\left(1-c_{1}\right) \mathrm{e}^{\left(2 r-\theta^{\top} \theta\right)(T-t)}}{1-c_{1} \mathrm{e}^{\left(2 r-\theta^{\top} \theta\right)(T-t)}}, \quad \forall t \in[0, T],
$$

with $c_{1}=1 /\left(-2 r+\theta^{\top} \theta+1\right)$.

The efficient strategies depend on the term $\gamma \mathrm{e}^{-r(T-t)}-X(t)$ that, by the definition of $\gamma$ in Proposition 3.1, decomposes in three terms that we collect into two summands

$$
\frac{1}{2 \mu} \mathrm{e}^{-r(T-t)}+\left[\mathbb{E}\left(X(T) \mathrm{e}^{-r(T-t)}\right)-X(t)\right] .
$$

The first summand is always positive, increasing with time, and depends inversely on $\mu$, the parameter weighing the relative importance of the objective of variance minimization with respect to the objective of debt reduction. The summand in brackets is the expected value of debt reduction planned, valued at time $t$. Notice from the expression of $S C^{*}$ that if this reduction is positive, then amortization rate is higher than the normal cost. In the same way, the first summand in $\Lambda^{*}$ is also positive. Of course, this behavior is also observed for small values of $\mu$, even if there is no reduction of the expected debt. As the control of variance becomes less important for the sponsor, that is, as $\mu$ decreases, the investment strategies are riskier.

In contradistinction to the supplementary cost, optimal investment depends also on $A L$ and on the elements giving the randomness of assets and benefits. If the actuarial liability $A L$ is positively correlated with the financial market (an extreme case being uncorrelated, where $q=0$ ), then the investment in the risky assets is greater than if the correlation is negative. It is also remarkable that does not depend on the rate of growth of benefits, $\kappa$.

A technical assumption to obtain some properties of the optimal solutions is necessary. We suppose that twice the risk-free rate of interest is lesser than the norm square of the Sharpe ratio.

Assumption 3. The Sharpe ratio vector satisfies $2 r<\theta^{\top} \theta$.

This hypothesis implies for the constant $c_{1}$ and the function $f$ defined in Theorem 4.1 that $0<c_{1}<1$ and $0<f(t)<1$, for all $0 \leqslant t<T$.

Theorem 4.1 gives also a linear relationship between the supplementary cost and investment strategies, which vector coefficient is the optimal growth portfolio, $\Sigma^{-1}(\mathbf{b}-r \mathbf{1})$, multiplied by the inverse of $f(t)$ :

$$
\mathbf{\Lambda}^{*}=\frac{1}{f(t)} \Sigma^{-1}(\mathbf{b}-r \mathbf{1}) S C^{*}+\eta \sigma^{-\top} q A L .
$$

This can be considered as a "rule of thumb" for the sponsor: at time $t$, each monetary unit of additional amortization with respect to the computed normal cost, must be accompanied by an investment of $\frac{1}{f(t)} \Sigma^{-1}(\mathbf{b}-r \mathbf{1})$ monetary units in risky assets, plus $\eta \sigma^{-\top} q A L$ units due to the stochastic elements defining the pension plan.

The following result characterizes the efficient frontier in terms of the expected returns and variance (disregarding the influence of the contribution risk).

Theorem 4.2. The mean variance efficient frontier of the problem (1), (9), (10) is given by

$$
\vee \operatorname{ar} X(T)=\left(\frac{1-\beta}{\beta}\right)^{2}\left(\mathrm{e}^{\theta^{\top} \theta T}-1\right)\left(\mathbb{E} X(T)-\mathrm{e}^{r T} X_{0}\right)^{2}+v,
$$


where

$$
\begin{aligned}
& \beta=1-\mathrm{e}^{-\theta^{\top} \theta T} \frac{1-c_{1}}{1-c_{1} \mathrm{e}^{\left(2 r-\theta^{\top} \theta\right) T}}=1-\mathrm{e}^{2 r T} f(0), \\
& v=\eta^{2}\left(1-q^{\top} q\right) A L_{0}^{2} \mathrm{e}^{\left(2 \kappa+\eta^{2}\right) T} \int_{0}^{T} \frac{\mathrm{e}^{\left(2 r-\theta^{\top} \theta-2 \kappa-\eta^{2}\right) t}}{\left(1-c_{1} \mathrm{e}^{\left(2 r-\theta^{\top} \theta\right) t}\right)^{2}} \mathrm{~d} t .
\end{aligned}
$$

Expression (17) shows the familiar quadratic relation between debt and its variance. The minimum possible variance, $\operatorname{Var} X(T)=v \geqslant 0$, is attained when the sponsor borrows money for the total amount of debt at date $t=0$ for $T$ years, so that $\mathbb{E} X(T)=\mathrm{e}^{r T} X_{0}$.

From (17), the expected debt and the standard deviation, $\sigma_{X}(T)$, at time $T$ are related by

$$
\mathbb{E} X(T)=\mathrm{e}^{r T} X_{0}+\frac{\beta}{(1-\beta) \sqrt{ } \mathrm{e}^{\theta^{\top} \theta T}-1} \sqrt{ } \sigma_{X}^{2}(T)-v .
$$

There are two cases where it is a straight line: when the benefits are exponential and deterministic, $\eta=0$, and when the market is complete (Brownian $B$ only depends on $w$ ), $q^{\top} q=1$. In both cases the capital market line is

$$
\mathbb{E} X(T)=\mathrm{e}^{r T} X_{0}+\frac{\beta}{(1-\beta) \sqrt{ } \mathrm{e}^{\theta^{\top} \theta T}-1} \sigma_{X}(T),
$$

The slope, $\beta /\left((1-\beta) \sqrt{ } \mathrm{e}^{\theta^{\top} \theta T}-1\right)$, is the price of risk. This is positive because $0<\beta<1$ by Assumption 3 . It shows how much the expected optimal debt decreases if its standard deviation increases by one unit.

Observe that parameter $v$ and in consequence the terminal variance in (17) does not depend on the sign of correlations $q_{i}$.

Remark 4.1. The optimal investment decisions, contribution rate and fund's wealth evolution can be expressed in terms of the optimal expected debt at time $T, \mathbb{E} X^{*}(T)$, instead of using the parameters $\gamma$ or $\mu$. This provides a more clever interpretation of the results. The substitution of $\gamma$ may be done from the equality $\mathrm{e}^{r T}(1-\beta) X_{0}+\beta \gamma=\mathbb{E} X^{*}(T)$, which is obtained in (29) in Appendix A. Taking into account (14) and (29), the investment at instant $t$ is

$$
\Lambda^{*}(t, X, A L)=\Sigma^{-1}(\mathbf{b}-r \mathbf{1})\left(\frac{\mathrm{e}^{-r(T-t)}}{\beta}\left(z-\mathrm{e}^{r T} X_{0}\right)-\left(X-\mathrm{e}^{r t} X_{0}\right)\right)+\eta \sigma^{-\top} q A L,
$$

where $z=\mathbb{E} X^{*}(T)$. This shows the existing relation between the desired expected levels of debt at time $T$ and the optimal composition of the portfolio at every instant of time $t$.

Analogously, (13) and (29), allows us to rewrite the optimal rate of contribution at instant $t$ as

$$
C^{*}(t, X)=N C(t)+f(t)\left(\frac{\mathrm{e}^{-r(T-t)}}{\beta}\left(z-\mathrm{e}^{r T} X_{0}\right)-\left(X-\mathrm{e}^{r t} X_{0}\right)\right) .
$$

The following proposition gives the total optimal contribution.

Proposition 4.1. The total expected discounted value of the optimal contribution and the optimal supplementary cost in the interval $[0, T]$ of problem (1), (9), (10), denoted $\bar{C}$ and $\overline{S C}$ respectively, are given by

$$
\begin{aligned}
& \overline{S C} \doteq \mathbb{E} \int_{0}^{T} \mathrm{e}^{-r t} S C^{*}(t, X(t)) \mathrm{d} t=\pi\left(\mathbb{E} X(T)-\mathrm{e}^{r T} X_{0}\right), \\
& \bar{C} \doteq \mathbb{E} \int_{0}^{T} \mathrm{e}^{-r t} N C(t) \mathrm{d} t+\overline{S C}=\frac{1-\mathrm{e}^{-(r-\kappa) T}}{r-\kappa} N C_{0}+\overline{S C},
\end{aligned}
$$

where

$$
\pi=\frac{1-\beta}{\beta} \frac{\mathrm{e}^{2 r T}-1}{2 r} \mathrm{e}^{-r T} .
$$


The relation between $\overline{S C}$ and $\mathbb{E} X(T)$ is linear, with positive slope $\pi$, since $0<\beta<1$ by Assumption 3 . Thus, a reduction of one monetary unit of expected debt at time $T$ is attained with an extra expected amortization of $\pi$ monetary units over the total expected discounted normal cost computed along the time horizon $[0, T]$.

Other interesting fact is that total contribution does not depend on diffusion parameter $\eta$, so it coincides with total contribution in the case of deterministic benefits.

Since the mixed portfolio has higher mean returns than the bond, the contribution effort made by the sponsor under risky investment is less than the contribution effort arising under safe investment. This is proved in the following result.

\section{Corollary 4.1}

(a) The total expected value of the supplementary cost is less when the manager invests in the mixed portfolio than when he or she invests only in the bond.

(b) If $q^{\top} \theta \geqslant 0$ then the total expected value of the optimal contribution is less when the manager invests in the mixed portfolio than when he or she invests only in the bond.

\section{A numerical illustration}

In this section we illustrate the results of section above in a specific example. In order to give a more sound illustration of the model's properties we consider two risky assets. The objective is to observe the behavior of the terminal standard deviation, the initial investment and the total expected optimal contribution, with respect to the terminal date, the expected terminal debt and the correlations between benefits and risky assets. The values of parameters that we consider are the following.

- benefits are random with $\eta=0.03$ and $\kappa=0.2$;

- the risk-free rate of interest is $r=0.06$; - risky investment is in two assets $(n=2)$ with $\mathbf{b}=(0.12,0.10)^{\top}$ and $\sigma=\left(\begin{array}{cc}0.15 & 0.07 \\ 0.07 & 0.10\end{array}\right)$; this implies a
Sharpe ratio $\theta=(0.317,0.178)^{\top}$;

- the initial values are $A L_{0}=1, F_{0}=0.8$, so that the initial liability is $X_{0}=-0.2$, that is, the fund is $20 \%$ underfunded; benefits at time $t=0$ are supposed to be $1 \%$ of $A L_{0}$, that is, $P_{0}=0.01$.

We consider four values of the time horizon, $T=1,2,5$ and 10 years. The goal of the manager is to reduce expected debt $\mathbb{E} X(T)$ to values $-0.15,-0.10,-0.05$ and 0 , that is to say, to attain the $25 \%, 50 \%, 75 \%$ and $100 \%$, of debt reduction, respectively. The last variable elements that we consider are the correlations $q=\left(q_{1}, q_{2}\right)^{\top}$. We suppose the norm square of correlation vector, $q^{\top} q=q_{1}^{2}+q_{2}^{2}$, takes the values $0,0.50$ and 1 . More values could be considered obtaining similar properties. The extreme values are uncorrelation and perfect correlation, respectively. In order to fix the correlations we suppose symmetric cases, i.e. $q_{1}= \pm q_{2}$, so the vectors $q$ considered are $(0,0)^{\top},( \pm 1 / 2, \pm 1 / 2)^{\top}$ and $( \pm \sqrt{ } 2 / 2, \pm \sqrt{ } 2 / 2)^{\top}$.

The remainder elements depending on correlations are easily found. For instance, the technical interest rate $\delta$ can be calculated from Assumption 2, and from this, Proposition 2.1 for $t=0$ allows to obtain $N C_{0}$ in each case.

Table 1 shows for several final dates of the pension plan what the terminal standard deviation must be in order to reduce debt. We observe that the standard deviation does not depend on the sign of the correlations

Table 1

Terminal standard deviation $\sigma_{X(T)}$

\begin{tabular}{|c|c|c|c|c|c|c|c|c|c|c|c|c|c|c|c|c|c|c|}
\hline \multirow[t]{2}{*}{ Expected debt $z$} & \multirow[t]{2}{*}{$\mathbb{E} X(T)$} & \multicolumn{4}{|c|}{ Uncorrelation $q^{\top} q$} & \multicolumn{6}{|c|}{ Intermediate correlation $q^{\top} q$} & \multicolumn{7}{|c|}{ Perfect correlation $q^{\top} q$} \\
\hline & & $T \quad 1$ & 2 & 5 & 10 & $T$ & 2 & $T$ & 5 & $T$ & 10 & $T$ & 1 & $T$ & 2 & $T$ & 5 & $T$ \\
\hline 0.15 & & 2.0029 & 2.7013 & 5.1545 & 14.1069 & 1.4163 & 1.9101 & 3.6 & & & 751 & 0.0 & 184 & & 144 & 0.0 & 112 & 0.0093 \\
\hline 0.05 & & 2.0034 & 2.7014 & 5.1546 & 14.1069 & 1.4170 & 1.9104 & 3.6 & & & 751 & 0.0 & 478 & 0.0 & 336 & 0.0 & 206 & 0.0137 \\
\hline 0 & & 2.0038 & 2.7016 & 5.1546 & 14.1069 & 1.4176 & 1.9105 & 3.6 & & & 751 & 0.0 & 626 & 0.0 & 431 & 0.0 & 253 & 0.0159 \\
\hline
\end{tabular}


and it grows with respect to the planning horizon when $q^{\top} q<1$, but it decreases when $q^{\top} q=1$. In fact, it is more sensible to changes in the horizon length than to changes in the reduction of debt.

Another interesting fact is that the standard deviation is reduced when the norm square of correlation vector is increased, attaining the minimum value when the market is complete.

The total amount of initial investment proportion in the risky assets, $\left(\lambda_{1}+\lambda_{2}\right) / F_{0}$, chosen to reduce debt to the prescribed levels, is shown in Table 2. The investment in the bond is $1-\left(\lambda_{1}+\lambda_{2}\right) / F_{0}$, that can be obtained from the table. There are two cases of shortselling, which appear of course when both correlations are the

Table 2

Initial investment in the risky assets $\left(\lambda_{1}\left(0, X_{0}, A L_{0}\right)+\lambda_{2}\left(0, X_{0}, A L_{0}\right)\right) / F_{0}$

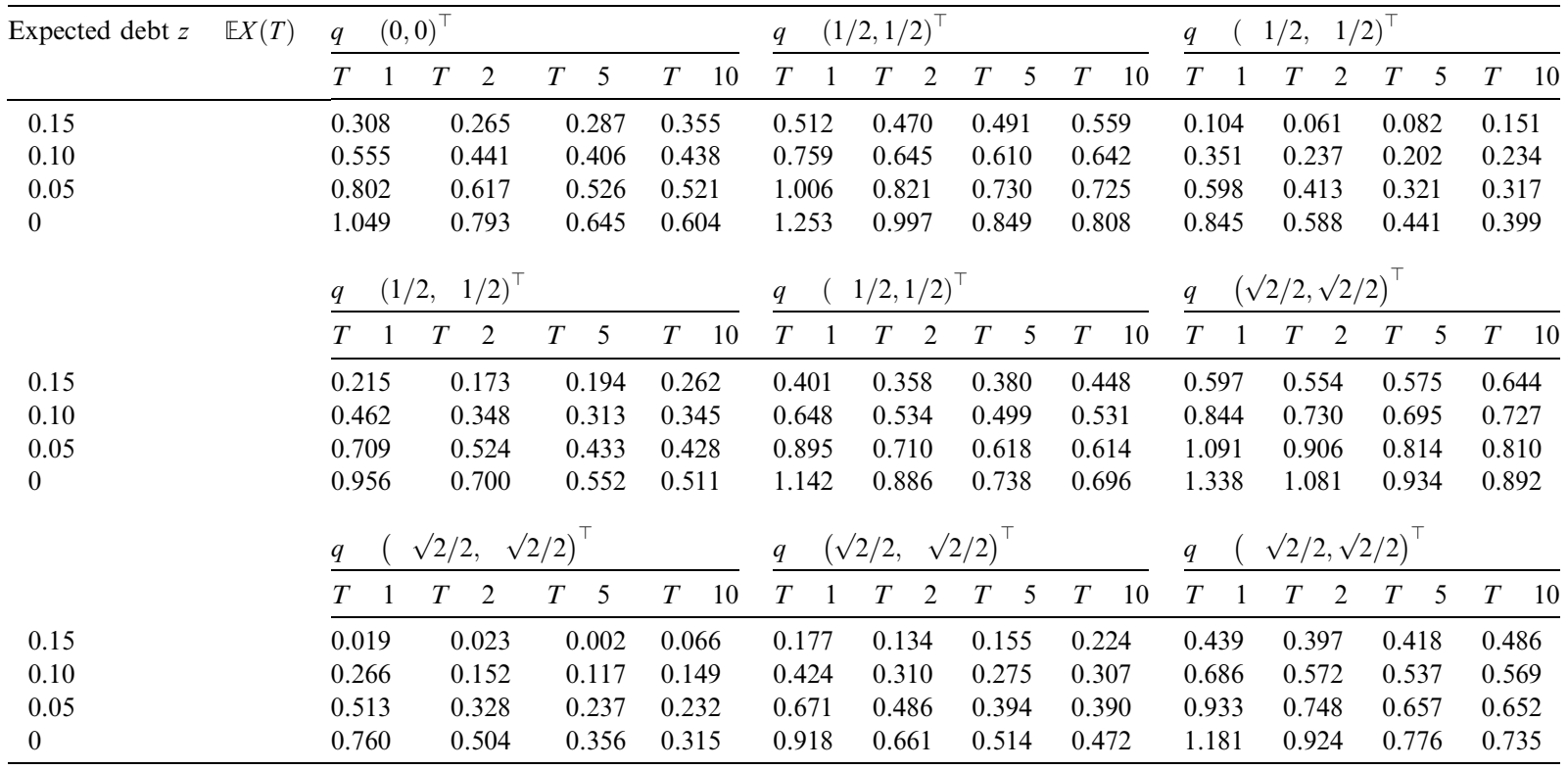

Table 3

Total expected discounted contribution $\bar{C}$

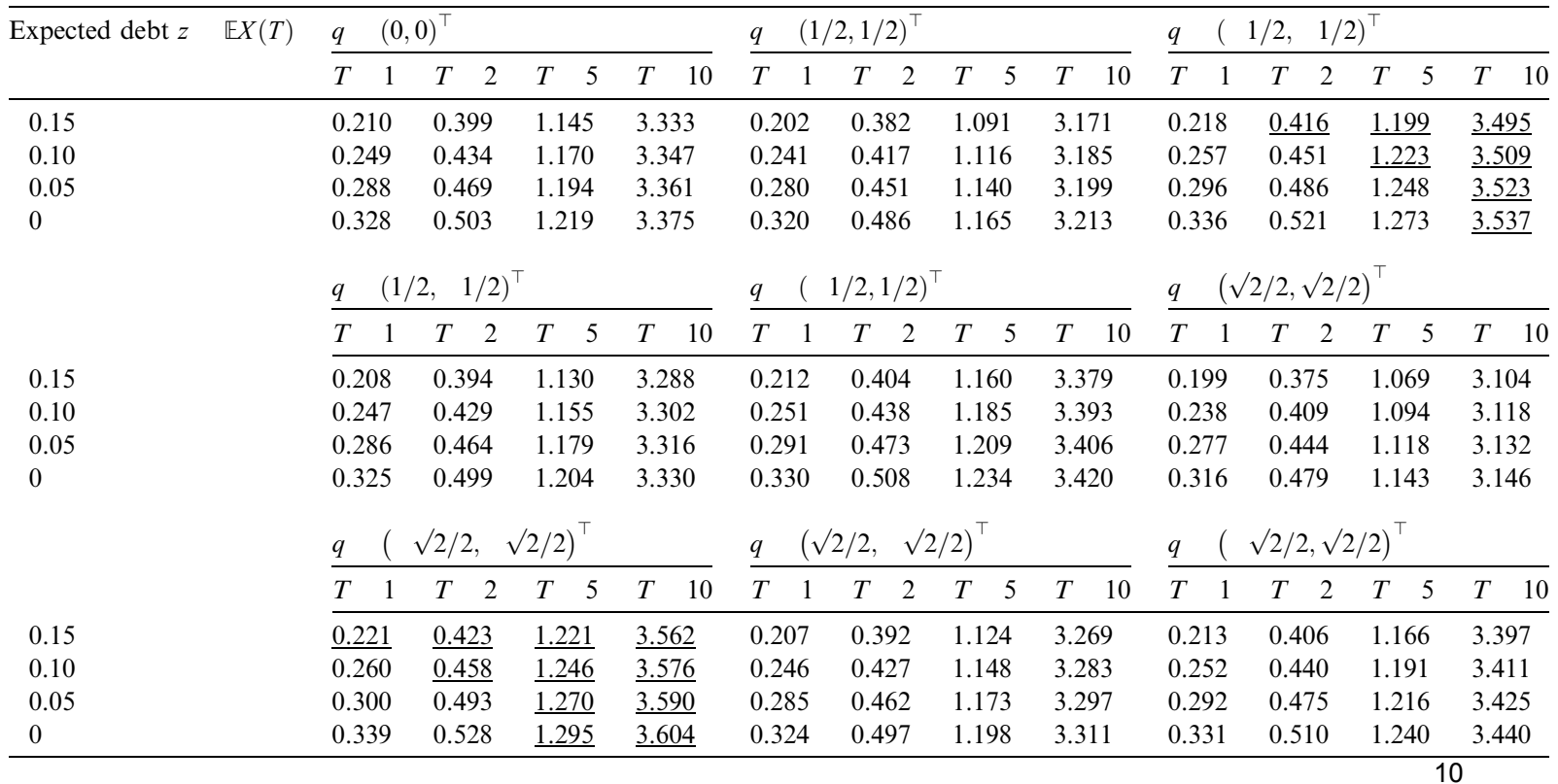


more negative allowed values. In some cases borrowing to invest in the risky assets is needed. Specially, this happens when the target is to eliminate completely debt in a short period of time. As expected, the risky investment increases with higher debt reduction levels. Note that there is a "horizon effect" in the investment strategies, since that they do not follow a monotonic pattern with respect to final time $T$.

The investment is sensible with respect to the sign of the correlations and the norm square of the correlations vector. With negative signs, a more conservative strategy is implemented and the investment behavior is more aggressive for higher levels of correlations.

Table 3 shows the total expected terminal optimal value of the contribution rate. The total contribution grows with the debt reduction and with the planning horizon. The correlation has little influence in the total contribution although it is smaller with positive correlation.

Table 4 shows the total expected optimal value of the supplementary cost when the portfolio comprises the bond and two risky assets, whereas Table 5 shows the result when investment is only in the bond. In both cases the supplementary cost grows with debt reduction, and a horizon effect appears as in the other components of the plan previously analyzed.

We observe in the tables the result obtained in Corollary 4.1: supplementary cost is greater when the investment is made only in the bond. Risky investment allows to get higher mean returns, making possible to diminish the expected amortization rate.

Table 6 is the corresponding one to Table 3, but showing the total expected contribution when the investment is in the bond only. When the inequality $q^{\top} \theta \geqslant 0$ holds, the total contribution with safe investment is higher than with investment in risky assets, see Corollary 4.1. When the opposite inequality holds, the property is not generally true. For example, in Table 3 are emphasized values where the optimal contributions with

Table 4

Total expected discounted supplementary cost

\begin{tabular}{lllllr}
\hline Expected debt $z$ & $\mathbb{E} X(T)$ & $\overline{S C}$ & & & \\
\cline { 2 - 6 } & $T \quad 1$ & $T \quad 2$ & 5 & 0.059 & 0.084 \\
\hline 0.15 & 0.049 & 0.053 & 0.087 & 0.108 & 0.074 \\
0.10 & 0.088 & 0.122 & 0.133 & 0.088 \\
0.05 & 0.127 & 0.157 & 0.102 \\
\hline
\end{tabular}

Table 5

Total expected discounted supplementary cost: safe investment

\begin{tabular}{lllllll}
\hline Expected debt $z$ & $\mathbb{E} X(T)$ & $\overline{S C}^{0}$ & & & \\
\cline { 2 - 6 } & $T \quad 1$ & $T \quad 2$ & $T$ & 5 \\
\hline 0.15 & 0.059 & 0.067 & 0.089 & 0.126 \\
0.10 & 0.106 & 0.111 & 0.163 & 0.145 \\
0.05 & 0.153 & 0.156 & 0.200 & 0.173 \\
0 & 0.200 & 0.200 & 0.200 \\
\hline
\end{tabular}

Table 6

Total expected discounted contribution: safe investment

\begin{tabular}{|c|c|c|c|c|c|}
\hline \multirow[t]{2}{*}{ Expected debt $z$} & \multirow[t]{2}{*}{$\mathbb{E} X(T)$} & \multicolumn{4}{|l|}{$\bar{C}^{0}$} \\
\hline & & T 1 & T 2 & $T \quad 5$ & $T \quad 10$ \\
\hline 0.15 & & 0.220 & 0.413 & 1.175 & 3.391 \\
\hline 0.05 & & 0.314 & 0.502 & 1.249 & 3.446 \\
\hline 0 & & 0.361 & 0.546 & 1.286 & 3.473 \\
\hline
\end{tabular}


investment in risky assets is greater than the corresponding with investment only in the bond; of course in all of them $q$ verifies $q^{\top} \theta<0$.

Finally, in all the cases of Tables 46 there is no dependency with respect to the correlations.

\section{Conclusions}

We have analyzed the management of a pension funding process of an aggregated defined benefit pension plan where the benefits are stochastic. The objective is to determine contributions and investments strategies maximizing the expected terminal fund and at the same time minimizing both the contribution risk and the variance of the unfunded actuarial liability. The problem is formulated as a modified mean variance optimization problem and has been solved by means of dynamic programming techniques.

The efficient frontier has a parabolic shape, but it is not given by a perfect square due to the randomness of benefits and to the existence of correlations between risky assets and benefits. The optimal investment strategies have three summands. One term depends on the current level of the actuarial liability, with a coefficient involving the instantaneous variance of benefits and risky assets, as well as its correlation. Other summand depends on the preferences of the sponsor, that is, of the relative importance of the objectives in the minimization functional. The remainder summand is proportional to the present expected value of debt reduction planned. We have also found what seems to be a new result in the literature of DB pension funds due to the stochastic character of the pension plan: there is a linear relationship between the optimal supplementary cost and the vector of optimal investment strategies, given in (16). A correction term is present due to the random behavior of benefits.

We have also proved that under suitable conditions about the sign of the correlations, the total expected contribution is lesser when the investment is in the mixed portfolio than when it is in the bond only.

A numerical illustration shows the analytical results proved in the paper, as well as other features of the model.

Further research should be directed to include: no-shortselling and no-borrowing restrictions, final bankruptcy prohibition, stochastic riskless rate of interest and other bi-objective problems.

\section{Acknowledgements}

Both authors gratefully acknowledge financial support from Regional Government of Castilla y León (Spain) under project VA099/04 and Spanish Ministry of Education and Science and FEDER funds under project MTM2005-06534. We are indebted to three anonymous referees for the criticisms and valuable comments.

\section{Appendix A}

Proof of Proposition 3.1. The proof relies in a standard separation argument for concave functions. It follows the arguments in Zhou and Li (2000), but we have an extra term in the form of the running cost giving the contribution risk. Let $\left(S C^{*}, \Lambda^{*}\right)$ be an optimal solution of problem (1), (9), (11), with associated process $X^{*}$, where $\gamma=\mu^{1} / 2+\mathbb{E} X^{*}(T)$. Let us suppose it is not optimal solution of (1), (9), (12). Then there exists an admissible strategy $(S C, \boldsymbol{\Lambda})$ such that the associated path $X$ verifies $J(S C, \boldsymbol{\Lambda})<J\left(S C^{*}, \boldsymbol{\Lambda}^{*}\right)$, that is to say,

$$
\mathbb{E} \int_{0}^{T} S C^{2}(t) \mathrm{d} t-\mathbb{E} \int_{0}^{T}\left(S C^{*}\right)^{2}(t) \mathrm{d} t+\mathbb{E} X^{2}(T)-\mathbb{E}\left(X^{*}\right)^{2}(T)-2 \gamma\left(\mathbb{E} X(T)-\mathbb{E} X^{*}(T)\right)<0 .
$$

The function $g\left(y_{1}, y_{2}, y_{3}\right)=\mu\left(y_{1}+y_{3}\right)-\mu y_{2}^{2}-y_{2}$ is concave in $\mathbb{R}^{3}$ because the Hessian matrix is negative semidefinite. Observe

$$
g\left(\mathbb{E} X^{2}(T), \mathbb{E} X(T), \mathbb{E} \int_{0}^{T} S C^{2}(t) \mathrm{d} t\right)=J_{1}(S C, \boldsymbol{\Lambda})+\mu J_{2}(S C, \boldsymbol{\Lambda}),
$$

which is the objective function of problem (1), (9), (11). 
The concavity ${ }^{4}$ of $g$ and (18) imply

$$
\begin{aligned}
g( & \left.\mathbb{E} X^{2}(T), \mathbb{E} X(T), \mathbb{E} \int_{0}^{T} S C^{2}(t) \mathrm{d} t\right) \\
\leqslant & g\left(\mathbb{E}\left(X^{*}\right)^{2}(T), \mathbb{E} X^{*}(T), \mathbb{E} \int_{0}^{T}\left(S C^{*}\right)^{2}(t) \mathrm{d} t\right)+\mu\left(\mathbb{E} X^{2}(T)-\mathbb{E}\left(X^{*}\right)^{2}(T)\right) \\
& -\left(1+2 \mu \mathbb{E} X^{*}(T)\right)\left(\mathbb{E} X(T)-\mathbb{E} X^{*}(T)\right)+\mu\left(\mathbb{E} \int_{0}^{T} S C^{2}(t) \mathrm{d} t-\mathbb{E} \int_{0}^{T}\left(S C^{*}\right)^{2}(t) \mathrm{d} t\right) \\
\leqslant & g\left(\mathbb{E}\left(X^{*}\right)^{2}(T), \mathbb{E} X^{*}(T), \mathbb{E} \int_{0}^{T}\left(S C^{*}\right)^{2}(t) \mathrm{d} t\right)+\mu\left(\mathbb{E} X^{2}(T)-\mathbb{E}\left(X^{*}\right)^{2}(T)-2 \gamma\left(\mathbb{E} X(T)-\mathbb{E} X^{*}(T)\right)\right. \\
& \left.+\mathbb{E} \int_{0}^{T} S C^{2}(t) \mathrm{d} t-\mathbb{E} \int_{0}^{T}\left(S C^{*}\right)^{2}(t) \mathrm{d} t\right)<g\left(\mathbb{E}\left(X^{*}\right)^{2}(T), \mathbb{E} X^{*}(T), \mathbb{E} \int_{0}^{T}\left(S C^{*}\right)^{2}(t) \mathrm{d} t\right) .
\end{aligned}
$$

Therefore $J_{1}\left(S C^{*}, \boldsymbol{\Lambda}^{*}\right)+\mu J_{2}(S C, \boldsymbol{\Lambda})<J_{1}(S C, \boldsymbol{\Lambda})+\mu J_{2}\left(S C^{*}, \boldsymbol{\Lambda}^{*}\right)$, by (19), that is to say, $\left(S C^{*}, \boldsymbol{\Lambda}^{*}\right)$ is not optimal for (1), (9), (11), which is a contradiction.

Proof of Theorem 4.1. In order to prove this result we use the dynamic programming approach, see Fleming and Soner (1993). Consider the value function of the control problem (1), (9), (12)

$$
\widehat{V}(t, X, A L)=\min _{(S C, \mathbf{\Lambda}) \in \mathscr{A}_{X, A L}}\{J((t, X, A L) ; S C, \boldsymbol{\Lambda}): \quad \text { s.t. (1), (9) }\} .
$$

It is well known $\widehat{V}$ is solution of the HJB equation:

$$
\begin{aligned}
& V_{t}+\min _{S C, A}\left\{S C^{2}+\left(r X+\boldsymbol{\Lambda}^{\top}(\mathbf{b}-r \mathbf{1})+S C-\eta q^{\top} \theta A L\right) V_{X}+\kappa A L V_{A L}\right. \\
& \left.\quad+\frac{1}{2}\left(\boldsymbol{\Lambda}^{\top} \Sigma \boldsymbol{\Lambda}-2 \eta A L \boldsymbol{\Lambda}^{\top} \sigma q+\eta^{2} A L\right) V_{X X}+\frac{1}{2} \eta^{2} A L^{2} V_{A L, A L}+\left(\eta A L \boldsymbol{\Lambda}^{\top} \sigma q-\eta^{2} A L^{2}\right) V_{X, A L}\right\}=0, \\
& V(T, X, A L)=X^{2}-2 \gamma X .
\end{aligned}
$$

Note that in (20) we have used (9) and the SDE of $A L$ as function of the Brownian motions $\left\{w_{i}\right\}_{i}^{n}$, obtained from (1), that is

$$
\mathrm{d} A L(t)=\kappa A L(t) \mathrm{d} t+\eta A L(t) \sqrt{ } 1-q^{\top} q \mathrm{~d} w_{0}(t)+\eta A L(t) q^{\top} \mathrm{d} w(t) .
$$

If there exists a smooth solution $V$ of this equation, strictly convex with respect to $X$, then the minimizer values of the supplementary cost and investments are given by

$$
\widehat{S C}\left(V_{X}\right)=-\frac{V_{X}}{2}, \quad \widehat{\boldsymbol{\Lambda}}\left(V_{X}, V_{X X}, V_{X, A L}\right)=-\Sigma^{-1}(\mathbf{b}-r \mathbf{1}) \frac{V_{X}}{V_{X X}}+\eta A L \sigma^{-\top} q\left(1-\frac{V_{X, A L}}{V_{X X}}\right) .
$$

After substitution of these values in (20) we obtain $\widehat{V}$ satisfies

$$
\begin{aligned}
V_{t} & +r X V_{X}-\frac{1}{4} V_{X}^{2}-\frac{1}{2} \theta^{\top} \theta \frac{V_{X}^{2}}{V_{X X}}+\kappa A L V_{A L}+\frac{1}{2} \eta^{2} A L^{2} V_{A L, A L}+\frac{1}{2} \eta^{2} A L^{2}\left(1-q^{\top} q\right) V_{X X}-\eta^{2} A L^{2}\left(1-q^{\top} q\right) V_{X, A L} \\
& -\eta A L \theta^{\top} q V_{X} \frac{V_{X, A L}}{V_{X X}}-\frac{1}{2} \eta^{2} A L^{2} q^{\top} q \frac{V_{X, A L}^{2}}{V_{X X}}=0
\end{aligned}
$$

with the final condition (21). We try a quadratic solution of the form

$$
\widehat{V}(t, X, A L)=\beta_{0}(t)+\beta_{X}(t) X+\beta_{A L}(t) A L+\beta_{X X}(t) X^{2}+\beta_{A L, A L}(t) A L^{2}+\beta_{X, A L}(t) X A L,
$$

\footnotetext{
${ }^{4}$ If $g: \mathbb{R}^{3} \rightarrow \mathbb{R}$ is a concave function of class $\mathscr{C}^{1}$, then $\forall \bar{x}, \bar{y} \in \mathbb{R}^{3}, g(\bar{x}) \quad g(\bar{y}) \leqslant \nabla g(\bar{y})(\bar{x} \quad \bar{y})$, where $\nabla g(\bar{y})$ denotes the gradient vector of $g$ at $\bar{y}$, i.e. $\nabla g(\bar{y}) \quad\left(g_{y_{1}}, g_{y_{2}}, g_{y_{3}}\right)$.
} 
so that from (22) the optimal controls must be

$$
\begin{aligned}
& \boldsymbol{\Lambda}=\Sigma^{-1}(\mathbf{b}-r \mathbf{1})\left(\frac{-\beta_{X}}{2 \beta_{X X}}-X-\frac{\beta_{X, A L}}{2 \beta_{X X}} A L\right)+\eta A L \sigma^{\top} q\left(1-\frac{\beta_{X, A L}}{\beta_{X X}}\right), \\
& S C=-\frac{1}{2}\left(\beta_{X}+2 \beta_{X X} X+\beta_{X, A L} A L\right)=\beta_{X X}\left(-\frac{\beta_{X}}{2 \beta_{X X}}-X-\frac{\beta_{X, A L}}{2 \beta_{X X}} A L\right) .
\end{aligned}
$$

The following ordinary differential equations are obtained for the above coefficients:

$$
\begin{aligned}
& \dot{\beta}_{0}=\frac{\beta_{X}^{2}}{4}+\frac{\theta^{\top} \theta}{4} \beta_{X}^{2} \beta_{X X}, \quad \beta_{0}(T)=0, \\
& \dot{\beta}_{X}=\left(-r+\theta^{\top} \theta\right) \beta_{X}+\beta_{X} \beta_{X X}, \quad \beta_{X}(T)=-2 \gamma, \\
& \dot{\beta}_{A L}=-\kappa \beta_{A L}+\frac{1}{2}\left(\theta^{\top} \theta+\eta \theta^{\top} q\right) \frac{\beta_{X} \beta_{X, A L}}{\beta_{X X}}+\frac{1}{2} \beta_{X} \beta_{X, A L}, \quad \beta_{A L}(T)=0, \\
& \dot{\beta}_{X X}=\left(-2 r+\theta^{\top} \theta\right) \beta_{X X}+\beta_{X X}^{2}, \quad \beta_{X X}(T)=1 . \\
& \dot{\beta}_{A L, A L}=-\left(2 \kappa+\eta^{2}\right) \beta_{A L, A L}+\left(\frac{\theta^{\top} \theta}{4}+\frac{\eta}{2} \theta^{\top} q+\eta^{2} q^{\top} q\right) \frac{\beta_{X, A L}^{2}}{\beta_{X X}} \\
& \quad+\eta^{2}\left(1-q^{\top} q\right)\left(\beta_{X, A L}-\beta_{X X}\right)+\frac{\beta_{X, A L}^{2}}{4}, \quad \beta_{A L, A L}(T)=0, \\
& \dot{\beta}_{X, A L}=\left(-r-\kappa+\eta+\theta^{\top} \theta\right) \beta_{X, A L}+\beta_{X X} \beta_{X, A L}, \quad \beta_{X, A L}(T)=0 .
\end{aligned}
$$

The method of resolution of this system is standard. The solution of Eq. (25), of Ricatti type, can be found for example in Kloeden and Platen (1999, p. 572),

$$
\beta_{X X}(t)=f(t),
$$

and using this explicit expression of $\beta_{X X}$ we can obtain from (24) (see Arnold (1974, p. 139))

$$
\beta_{X}(t)=-2 \gamma \mathrm{e}^{-r(T-t)} f(t) .
$$

Substituting in (26), $\beta_{X A L}$ is given by

$$
\dot{\beta}_{X, A L}=\left(-r-\kappa+\eta+\theta^{\top} \theta+f(t)\right) \beta_{X, A L}, \quad \beta_{X, A L}(T)=0,
$$

that is to say $\beta_{X, A L}=0$. Plugging these expressions into (23) we obtain (13) and (14), respectively.

Proof of Theorem 4.2. Under the optimal feedback control (13), (14), the stochastic differential equation for process $X,(9)$, is

$$
\begin{aligned}
\mathrm{d} X(t)= & \left(\left(r-\theta^{\top} \theta-f(t)\right) X(t)+\left(\theta^{\top} \theta+f(t)\right) \gamma \mathrm{e}^{-r(T-t)}\right) \mathrm{d} t-\eta \sqrt{ } 1-q^{\top} q A L(t) \mathrm{d} w_{0}(t) \\
& +\theta^{\top}\left(\gamma \mathrm{e}^{-r(T-t)}-X(t)\right) \mathrm{d} w(t),
\end{aligned}
$$

with $X(0)=X_{0}$. Applying the Ito's formula to $X^{2}$ we obtain

$$
\begin{aligned}
\mathrm{d} X^{2}(t)= & 2\left(\left(r-\theta^{\top} \theta / 2-f(t)\right) X^{2}(t)+f(t) \gamma \mathrm{e}^{-r(T-t)} X(t)+(1 / 2) \theta^{\top} \theta \gamma^{2} \mathrm{e}^{-2 r(T-t)}\right. \\
& \left.+(1 / 2) \eta^{2}\left(1-q^{\top} q\right) A L^{2}(t)\right) \mathrm{d} t-2 \eta \sqrt{ } 1-q^{\top} q A L(t) X(t) \mathrm{d} w_{0}(t) \\
& +2 \theta^{\top}\left(\gamma \mathrm{e}^{-r(T-t)} X(t)-X^{2}(t)\right) \mathrm{d} w(t),
\end{aligned}
$$

with $X^{2}(0)=X_{0}^{2}$. Taking expectations on both previous stochastic differential equations we obtain that functions $m_{1}(t)=\mathbb{E} X(t)$ and $m_{2}(t)=\mathbb{E} X^{2}(t)$ satisfy the linear ordinary differential equations

$$
\begin{aligned}
& \dot{m}_{1}(t)=\left(r-\theta^{\top} \theta-f(t)\right) m_{1}(t)+\left(\theta^{\top} \theta+f(t)\right) \gamma \mathrm{e}^{-r(T-t)}, \quad m_{1}(0)=X_{0}, \\
& \dot{m}_{2}(t)=\left(2 r-\theta^{\top} \theta-2 f(t)\right) m_{2}(t)+2 f(t) \gamma \mathrm{e}^{-r(T-t)} m_{1}(t)+\theta^{\top} \theta \gamma^{2} \mathrm{e}^{-2 r(T-t)}+\eta^{2}\left(1-q^{\top} q\right) A L_{0}^{2} \mathrm{e}^{\left(2 \kappa+\eta^{2}\right) t}, \quad m_{2}(0)=X_{0}^{2},
\end{aligned}
$$

where in (27) we have used that $\mathbb{E} A L^{2}(t)=A L_{0}^{2} \mathrm{e}^{\left(2 \kappa+\eta^{2}\right) t}$, by (1). 
Following Arnold (1974, p. 139),

$$
m_{1}(t)=\mathbb{E} X(t)=\mathrm{e}^{\int_{0}^{t}\left(r-\theta^{\top} \theta-f(s)\right) \mathrm{d} s}\left(X_{0}+\left(\theta^{\top} \theta+f(t)\right) \gamma \int_{0}^{t} \mathrm{e}^{-\int_{0}^{s}\left(r-\theta^{\top} \theta-f(v)\right) \mathrm{d} v} \mathrm{e}^{-r(T-s)} \mathrm{d} s\right)
$$

that, after some calculations it is

$$
\mathbb{E} X(t)=\mathrm{e}^{\left(r-\theta^{\top} \theta\right) t}\left(1-c_{1} \mathrm{e}^{\left(2 r-\theta^{\top} \theta\right)(T-t)}\right)\left(\frac{X_{0}}{1-c_{1} \mathrm{e}^{\left(2 r-\theta^{\top} \theta\right) T}}+\gamma \mathrm{e}^{-\left(r-\theta^{\top} \theta\right) T}\left(\frac{\mathrm{e}^{-\theta^{\top} \theta(T-t)}}{1-c_{1} \mathrm{e}^{\left(2 r-\theta^{\top} \theta\right)(T-t)}}-\frac{\mathrm{e}^{-\theta^{\top} \theta T}}{1-c_{1} \mathrm{e}^{\left(2 r-\theta^{\top} \theta\right) T}}\right)\right),
$$

for all $t \in[0, T]$. For $t=T$ we have

$$
\mathbb{E} X(T)=\alpha X_{0}+\beta \gamma
$$

where

$$
\begin{aligned}
& \beta=1-\mathrm{e}^{-\theta^{\top} \theta T} \frac{1-c_{1}}{1-c_{1} \mathrm{e}^{\left(2 r-\theta^{\top} \theta\right) T}}=1-\mathrm{e}^{2 r T} f(0), \\
& \alpha=\mathrm{e}^{r T}(1-\beta) .
\end{aligned}
$$

Analogously,

$$
\begin{aligned}
m_{2}(t)= & \mathbb{E} X^{2}(t)=\mathrm{e}^{\int_{0}^{t}\left(2 r-\theta^{\top} \theta-2 f(s)\right) \mathrm{d} s}\left(X_{0}^{2}+2 \gamma \int_{0}^{t} \mathrm{e}^{-\int_{0}^{s}\left(2 r-\theta^{\top} \theta-2 f(v)\right) \mathrm{d} v} f(s) \mathrm{e}^{-r(T-s)} m_{1}(s) \mathrm{d} s\right. \\
& \left.+\theta^{\top} \theta \gamma^{2} \int_{0}^{t} \mathrm{e}^{-\int_{0}^{s}\left(2 r-\theta^{\top} \theta-2 f(v)\right) \mathrm{d} v} \mathrm{e}^{-2 r(T-s)} \mathrm{d} s+\eta^{2}\left(1-q^{\top} q\right) A L_{0}^{2} \int_{0}^{t} e^{-\int_{0}^{s}\left(2 r-\theta^{\top} \theta-2 f(v)\right) \mathrm{d} v} \mathrm{e}^{\left(2 \kappa+\eta^{2}\right) s} \mathrm{~d} s\right),
\end{aligned}
$$

that, after some calculations it is transformed in

$$
\mathbb{E} X^{2}(T)=\rho X_{0}^{2}+2 \alpha \gamma \epsilon X_{0}+\gamma^{2}(\beta-(1-\beta) \epsilon)+v,
$$

where

$$
\begin{aligned}
& \rho=\alpha^{2} \mathrm{e}^{\theta^{\top} \theta T}, \\
& \epsilon=1-(1-\beta) \mathrm{e}^{\theta^{\top} \theta T} \\
& v=\eta^{2}\left(1-q^{\top} q\right) A L_{0}^{2} \mathrm{e}^{\left(2 \kappa+\eta^{2}\right) T} \int_{0}^{T} \frac{\mathrm{e}^{\left(2 r-\theta^{\top} \theta-2 \kappa-\eta^{2}\right) t}}{\left(1-c_{1} \mathrm{e}^{\left(2 r-\theta^{\top} \theta\right) t}\right)^{2}} \mathrm{~d} t .
\end{aligned}
$$

In order to find the mean variance efficient frontier we obtain the terminal variance:

$$
\begin{aligned}
\mathbb{V} \operatorname{ar} X(T) & =\mathbb{E} X^{2}(T)-(\mathbb{E} X(T))^{2} \\
& =\rho X_{0}^{2}+2 \alpha \gamma \epsilon X_{0}+\gamma^{2}(\beta-(1-\beta) \epsilon)+v-(\mathbb{E} X(T))^{2} \\
& =\rho X_{0}^{2}+2 \alpha \frac{1}{\beta}\left(\mathbb{E} X(T)-\alpha X_{0}\right) \epsilon X_{0}+\frac{1}{\beta^{2}}\left(\mathbb{E} X(T)-\alpha X_{0}\right)^{2}(\beta-(1-\beta) \epsilon)+v-(\mathbb{E} X(T))^{2} \\
& =\frac{1-\beta}{\beta} \frac{\beta-\epsilon}{\beta}\left(\mathbb{E} X(T)-\mathrm{e}^{r T} X_{0}\right)^{2}+v \\
& =\left(\frac{1-\beta}{\beta}\right)^{2}\left(\mathrm{e}^{\theta^{\top} \theta T}-1\right)\left(\mathbb{E} X(T)-\mathrm{e}^{r T} X_{0}\right)^{2}+v
\end{aligned}
$$

where in the second equality we have used (30) and in the third one we have used (29). 
Proof of Proposition 4.1. By (13),

$$
\begin{aligned}
\mathbb{E} \int_{0}^{T} \mathrm{e}^{-r t} S C^{*}(t, X(t)) \mathrm{d} t & =\int_{0}^{T} \mathrm{e}^{-r t} f(t)\left(\gamma \mathrm{e}^{-r(T-t)}-\mathbb{E} X(T)\right) \mathrm{d} t \\
& =\frac{\left(1-c_{1}\right) \mathrm{e}^{-\theta^{\top} \theta T}}{1-c_{1} \mathrm{e}^{\left(2 r-\theta^{\top} \theta\right) T}}\left(\gamma \mathrm{e}^{-r T}-X_{0}\right) \int_{0}^{T} \mathrm{e}^{2 r t} \mathrm{~d} t \\
& =\pi\left(\mathbb{E} X(T)-\mathrm{e}^{r T} X_{0}\right),
\end{aligned}
$$

where the second equality is due to (15) and (28), and the third to the definition of $\beta$ and (29).

Proof of Corollary 4.1. We suppose that the manager wishes to attain an expected terminal debt $\mathbb{E} X(T)=z$ with both investment possibilities, in the mixed portfolio and in the fixed rent.

(a) Consider the total supplementary cost as a function of the Sharpe ratio of the portfolio, $\theta^{\top} \theta$ :

$$
\overline{S C}(y)=\frac{\mathrm{e}^{2 r T}-1}{2 r} \mathrm{e}^{-r T}\left(z-\mathrm{e}^{r T} X_{0}\right)\left(\frac{1}{\beta(y)}-1\right), \quad y \in[0, T],
$$

where

$$
\beta(y)=1-\mathrm{e}^{-T y} \frac{1-c_{1}(y)}{1-c_{1}(y) \mathrm{e}^{-T(-2 r+y)}},
$$

with $c_{1}(y)=1 /(-2 r+y+1)$. The expected total supplementary cost in the first situation is $\overline{S C}\left(\theta^{\top} \theta\right)$ and in the second $\overline{S C}(0)$.

It is very easy to check

$$
\beta^{\prime}(y)=T \mathrm{e}^{-T y} \frac{(-2 r+y)^{2}+(-2 r+y)+\mathrm{e}^{-(-2 r+y) T} / T}{\left(-2 r+y+1-\mathrm{e}^{-(-2 r+y) T}\right)^{2}}>0,
$$

by Assumption 3. Using (29) and Proposition 3.1 we obtain $z=\mathrm{e}^{r T} X_{0}+\beta /(2 \mu(1-\beta))>\mathrm{e}^{r T} X_{0}$, again by Assumption 3; that is, the expected terminal unfunded liability is lesser than the debt accrued at $t=T$ for borrowing money at date $t=0$ at an interest rate $r$. Thus we obtain $\overline{S C}^{\prime}(y)<0$, that is to say, $\overline{S C}$ is a strictly decreasing function in $\mathbb{R}$. Therefore $\overline{S C}\left(\theta^{\top} \theta\right)<\overline{S C}(0)$.

(b) The expected total contribution is in the first case

$$
\bar{C}=\frac{1-\mathrm{e}^{-(r-\kappa) T}}{r-\kappa} N C_{0}+\overline{S C}\left(\theta^{\top} \theta\right) .
$$

By Proposition 2.1 and Assumption 2, $N C_{0}=P_{0}+\left(\kappa-r-\eta q^{\top} \theta\right) A L_{0}$, that is smaller or equal to $P_{0}+(\kappa-r) A L_{0}$, because $q^{\top} \theta \geqslant 0$. Total contribution in the second case is obtained making $\theta=0$, so the proof finishes applying (a).

Justification of Assumption 2. Consider for the moment that only one worker exists, with age $x$. Once the liability is valued for one worker, the aggregated case is easily obtained as it is shown below. The sponsor wishes to value at the current time $t$ the asset $Y^{x}(t, P)$ consisting in paying $P$ monetary units at the age of retirement $d$, where $P$ is a geometric Brownian motion according to Assumption 1. Since $P$ is not tradeable and we suppose the existence of two independent sources of uncertainty, to value $Y^{x}$ we resort to equilibrium arguments, following the approach of Constantinides (1978). To simplify the exposition, let us consider that only a risky asset $S$ exists, which is freely traded in the market. The multidimensional case is straightforward. The method considers that the risk uncorrelated with $S$ is not priced. Consider the asset at any intermediate time, $Y^{x}(t+\tau, P), 0 \leqslant \tau \leqslant d-x$. Forming a portfolio with one unit of asset $Y^{x}$ and $\xi$ units of $S, R=Y^{x}+\xi S$, and applying Itô's Lemma, we have 


$$
\begin{aligned}
\mathrm{d} R & =\mathrm{d} Y^{x}+\xi \mathrm{d} S \\
& =\left(Y_{p}^{x} \kappa P+\frac{1}{2} Y_{p p}^{x} \eta^{2} P^{2}+Y_{\tau}^{x}+\xi b S\right) \mathrm{d} t+Y_{p}^{x} \eta P \mathrm{~d} B+\xi \sigma S \mathrm{~d} w \\
& =\left(Y_{p}^{x} \kappa P+\frac{1}{2} Y_{p p}^{x} \eta^{2} P^{2}+Y_{\tau}^{x}+\xi b S\right) \mathrm{d} t+Y_{p}^{x} \eta P \sqrt{ } 1-q^{2} \mathrm{~d} w_{0}+\left(Y_{p}^{x} \eta P q+\xi \sigma S\right) \mathrm{d} w .
\end{aligned}
$$

The first equality is the self-financing condition, the second one follows from Itô's Lemma, using that $P$ is geometric Brownian motion, and the last equality uses $B=\sqrt{ } 1-q^{2} w_{0}+q w$. The selection $\xi=-Y_{p}^{x} \eta P q / \sigma S$ eliminates the risk related with Brownian $w$. We also disregard the risk orthogonal to it, that is, the risk related with $w_{0}$ is not priced. The total return of the hedge portfolio must be equal to the risk-free rate of interest, $r\left(Y^{x}+\xi S\right)$. Thus we obtain the pricing partial differential equation

$$
r Y^{x}=Y_{\tau}^{x}+Y_{p}^{x} P\left(\kappa-\frac{q \eta}{\sigma}(b-r)\right)+\frac{1}{2} Y_{p p}^{x} \eta^{2} P^{2}
$$

with boundary conditions $Y^{x}(t+d-x, P)=P, Y^{x}(t+\tau, 0)=0$. The solution is

$$
Y^{x}(t+\tau, P)=P \mathrm{e}^{-(r-\kappa+q \eta \theta)(d-x-\tau)},
$$

hence at time of valuation $t(\tau=0), Y^{x}(t, P(t))=P(t) \mathrm{e}^{-(r-\kappa+q \eta \theta)(d-x)}$. Now, to obtain the total liability $A L(t)$ we aggregate the result for any age $x \in[a, d]$ having into account the way benefits accumulates depending on age, to obtain

$$
A L(t)=\int_{a}^{d} Y^{x}(t, P(t)) M(x) \mathrm{d} x=P(t) \int_{a}^{d} \mathrm{e}^{-(r-\kappa+q \eta \theta)(d-x)} M(x) \mathrm{d} x .
$$

On the other hand, the actuarial definition of $A L$ given in Section 2 and Assumption 1 provide

$$
A L(t)=P(t) \int_{a}^{d} \mathrm{e}^{-(\delta-\kappa)(d-x)} M(x) \mathrm{d} x .
$$

See the proof of Proposition 2.1 in Josa-Fombellida and Rincón-Zapatero (2004). Comparing the expressions obtained, we conclude that $\delta$ must be chosen equal to $r+q \eta \theta$ in order to attain a risk-neutral valuation of the liabilities.

Obtaining Eq. (9). Eq. (8) in terms of $X=F-A L$ and of $S C=C-N C$ is, by (1),

$$
\mathrm{d} X(t)=\left(r F(t)+\mathbf{\Lambda}^{\top}(t)(\mathbf{b}-r \mathbf{1})+S C(t)+N C(t)-P(t)-\kappa A L(t)\right) \mathrm{d} t+\boldsymbol{\Lambda}^{\top}(t) \sigma \mathrm{d} w(t)-\eta A L(t) \mathrm{d} B(t) .
$$

By Proposition 2.1 and Assumption 2, the above can be written

$$
\mathrm{d} X(t)=\left(r X(t)+\boldsymbol{\Lambda}^{\top}(t)(\mathbf{b}-r \mathbf{1})+S C(t)-\eta q^{\top} \theta A L(t)\right) \mathrm{d} t+\boldsymbol{\Lambda}^{\top}(t) \sigma \mathrm{d} w(t)-\eta A L(t) \mathrm{d} B(t),
$$

and, using the independent Brownian motions $\left\{w_{i}\right\}_{i}^{n}$, SDE (9) is obtained.

\section{References}

Arnold, L., 1974. Stochastic Differential Equations. Theory and Applications. John Wiley and Sons, New York.

Bowers, N.L., Gerber, H.U., Hickman, J.C., Jones, D.A., Nesbitt, 1986. Actuarial Mathematics. The Society of Actuaries, Itaca.

Cairns, A.J.G., 2000. Some notes on the dynamics and optimal control of stochastic pension fund models in continuous time. Astin Bulletin 30, 1955.

Chang, S.C., 1999. Optimal pension funding through dynamic simulations: The case of Taiwan public employees retirement system. Insurance: Mathematics and Economics 24, 187199.

Chang, S.C., Tsai, C.H., Tien, C.J., Tu, C.Y., 2002. Dynamic funding and investment strategy for defined benefit pension schemes: A model incorporating asset liability matching criteria. Journal of Actuarial Practice 10, 131153.

Chiu, M.C., Li, D., 2006. Asset and liability management under continuous time mean variance optimization framework. Insurance: Mathematics and Economics 39, 330355. 
Colombo, L., Haberman, S., 2005. Optimal contributions in a defined benefit pension scheme with stochastic new entrants. Insurance: Mathematics and Economics 37, 335354.

Constantinides, G., 1978. Market risk adjustment and project valuation. Journal of Finance 33, 603616.

Da Cunha, N.O., Polak, E., 1967. Constrained minimization under vector valued criteria in finite dimensional spaces. Journal of Mathematical Analysis and Applications 19, 103124.

Fleming, W.H., Soner, H.M., 1993. Controlled Markov Processes and Viscosity Solutions. Springer Verlag, New York.

Haberman, S., Sung, J.H., 1994. Dynamics approaches to pension funding. Insurance: Mathematics and Economics 15, 151162.

Haberman, S., Butt, Z., Megaloudi, C., 2000. Contribution and solvency risk in a defined benefit pension scheme. Insurance: Mathematics and Economics 27, 237259.

Huang, H.C., Cairns, A.J.G., 2005. On the control of defined benefit pension plans. Insurance: Mathematics and Economics $38,113131$.

Josa Fombellida, R., Rincón Zapatero, J.P., 2001. Minimization of risks in pension funding by means of contribution and portfolio selection. Insurance: Mathematics and Economics 29, 3545.

Josa Fombellida, R., Rincón Zapatero, J.P., 2004. Optimal risk management in defined benefit stochastic pension funds. Insurance: Mathematics and Economics 34, 489503.

Josa Fombellida, R., Rincón Zapatero, J.P., 2006a. Optimal investment decisions with a liability: The case of defined benefit pension plans. Insurance: Mathematics and Economics 39, 8198.

Josa Fombellida, R., Rincón Zapatero, J.P., 2006b. Funding and investment decisions in a stochastic defined benefit pension plan with several levels of labor income earnings. Computers and Operations Research, in press. doi:10.1016/j.cor.2006.02.021.

Kloeden, P.E., Platen, E., 1999. Numerical Solution of Stochastic Differential Equations. Springer, Berlin.

Li, D., Ng, W.L., 2000. Optimal dynamic portfolio selection: Multi period mean variance formulation. Mathematical Finance 10,387 406.

Markowitz, H.M., 1952. Portfolio selection. Journal of Finance 7, 7791.

Merton, R.C., 1971. Optimal consumption and portfolio rules in a continuous time model. Journal of Economic Theory 3 , 373413.

Merton, R.C., 1990. Continuous Time Finance. Blackwell, MA.

Taylor, G., 2002. Stochastic control of funding systems. Insurance: Mathematics and Economics 30, 323350.

Zhou, X.Y., Li, D., 2000. Continuous time mean variance portfolio selection: A stochastic LQ framework. Applied Mathematics and Optimization 42, 1933. 